\title{
O CUSTEIO ABC PARA FORMAÇÃO DO PREÇO DE VENDA EM UM LABORATÓRIO DE ANÁLISES FÍSICO-QUÍMICAS PARA ESTUDOS AMBIENTAIS
}

\author{
Renan Rozeng ${ }^{1}$, Eduardo Tramontin Castanha², Januario José Monteiro ${ }^{3}$, \\ Carolina Citadin Milaneze ${ }^{4}$, Andréia Cittadin ${ }^{5}$ \\ ${ }^{1}$ renan.rozeng@gmail.com \\ eduardo tramontin@unesc.net \\ ${ }^{3}$ januariomonteiromonteiro@gmail.com \\ ${ }^{4}$ Carolcmilaneze@hotmail.com \\ 5 aci@unesc.net
}

\section{Resumo}

O objetivo geral é apresentar os resultados de um estudo de caso que analisou a utilização do custeio ABC para a formação de preço de venda na prestação de serviços de um laboratório de análises físico-químicas para estudos ambientais. Para tanto, se fez uma pesquisa descritiva com abordagem qualitativa, mediante estudo de caso, pesquisa participante e documental com base em demonstrativos de resultado, inventários de imobilizados e estudos das demandas e horas técnicas dos serviços prestados. Os resultados apontaram que: a) a prestação de serviços nesse laboratório apresentou gastos indiretos na razão de $86 \%$ dos recursos totais; b) os recursos mais representativos referem-se à mão de obra, que correspondeu a 85\% dos gastos totais; c) a atividade que apresentou maiores custos foi planejar as amostragens; e d) o serviço que tem custo mais elevado é o Monitoramento de PTS (Partículas Totais em Suspensão). Os preços de vendas praticados pelo laboratório referente aos serviços da Matriz 1 - análises de emissões atmosféricas em dutos e chaminés, são superiores aos calculados com base no custeio ABC, com exceção para o serviço de análise de determinação de CO (Monóxido de Carbono). Os preços de vendas praticados para os serviços da Matriz 2- monitoramento da qualidade do ar em nível do solo, exceto para a avaliação de ruído, ficaram abaixo dos valores sugeridos no estudo. Conclui-se que esse laboratório tem seus maiores gastos relacionados à mão de obra e que o método de custeio $\mathrm{ABC}$ é uma ferramenta adequada para o reconhecimento dos custos nesse segmento e contribui para o estabelecimento do preço de venda.

Palavras Chave: Gestão de Custos. Método de Custeio ABC. Laboratório de Análises FísicoQuímicas para Estudos Ambientais. Formação de Preço.

\section{THE ABC COST FOR TRADING THE PRICE OF SALE IN A LABORATORY OF PHYSICAL-CHEMICAL ANALYSIS FOR ENVIRONMENTAL STUDIES}

\begin{abstract}
The general objective is to present the results of a case study that analyzed the use of $A B C$ costing for the formation of sale price in the provision of services of a laboratory of physicalchemical analysis for environmental studies. To do so, a descriptive research was carried out with a qualitative approach, through a case study, participatory and documentary research based on income statements, inventories of fixed assets and studies of the demands and technical hours of the services rendered. The results showed that: a) the services rendered in this laboratory presented indirect costs in the ratio of $86 \%$ of total resources; $b$ ) the most representative resources refer to the labor force, which corresponded to $85 \%$ of total expenditures; c) the activity that presented the highest costs was to plan the samplings; and d)
\end{abstract}


the service that has the highest cost is the PTS (Total Suspended Particle) Monitoring. The laboratory sales prices related to the services of Matrix 1 - analyzes of atmospheric emissions in ducts and chimneys, are higher than those calculated based on the ABC cost, except for the analysis service for the determination of $C O$ (Carbon Monoxide). The sales prices practiced for the services of Matrix 2 - air quality monitoring at ground level, except for the noise evaluation, were below the values suggested in the study. It is concluded that this laboratory has its highest costs related to labor and that the ABC costing method is an adequate tool for the recognition of costs in this segment and contributes to the establishment of the sale price.

Keywords: Costs management. ABC Costing Method. Laboratory of Physical-Chemical Analyzes for Environmental Studies. Pricing.

\section{Introdução}

Para as empresas se manterem competitivas em seu ramo de atuação é fundamental planejar e controlar os gastos e demais variáveis que compõem o preço do produto ou serviço ofertados. Entre os principais objetivos da contabilidade de custos está o controle dos gastos e o fornecimento de informações gerenciais que influenciam diretamente no processo de decisão das empresas, contribuindo na manutenção de uma entidade competitiva no mercado de atuação. Segundo Santos (2011) é visível que as organizações que não realizam a apuração e o gerenciamento dos custos e resultados, na atualidade, se baseando apenas no preço praticado pelo mercado, têm a tendência de insucesso em seus negócios.

$\mathrm{Na}$ busca de atender essas necessidades, o método de custeio ABC tem a capacidade de gerar informações detalhadas para a formação de preço de venda e subsidiar o gerenciamento dos recursos consumidos em cada atividade, no intuito de reduzir possíveis onerosidades no processo produtivo de uma organização.

$\mathrm{O}$ ABC se destaca nas organizações, por ser o método que possibilita fazer o cálculo dos custos indiretos com mais exatidão, pois agrega os gastos das áreas de vendas, transportes, distribuição e logística. Sua diferenciação em relação aos outros métodos de custeio é o tratamento em relação aos custos indiretos (NAKAGAWA, 2001). Martins (2003) salienta que o ABC tem como objetivo os processos e, a partir destes, a empresa evidencia quais atividades incluir no custeio. Para Ferreira (2016), essa metodologia diminui as distorções causadas pelos outros métodos de custeio, sobretudo em relação aos custos indiretos, fornecendo informações mais precisas sobre os custos dos produtos.

Contudo, é comum encontrar empresas que iniciam e conduzem suas atividades sem o devido planejamento, sendo definido o preço dos serviços prestados apenas pelo mercado, que muitas vezes não seguem uma forma coerente, propostas pelos métodos de custeio (SERRATINE; RAUPP, 2013). Desse modo, deixam de considerar os elementos que possam impactar no preço de venda, fator que pode afetar diretamente os seus resultados e diminuir o potencial de competividade.

O segmento de prestação de serviço de laboratórios de análises físico-químicas para estudos ambientais, como outros similares, depara-se com dificuldades como: (1) o repasse do aumento sobre preço do serviço prestado referente a reajustes inflacionários; (2) redução da demanda do mercado, por consequência do aumento da concorrência; (3) e a crise econômica que afeta seus clientes.

Essa situação torna-se mais um agravante em uma instituição que não possui como atividade fim somente esse tipo de serviço, pois o foco da gestão contempla outras áreas de atuação. Dessa forma, muitas vezes deixa-se de desenvolver estratégias para gerar receitas e obter melhores resultados. Diante disso, surge a necessidade de identificar o real consumo de 
recursos pelas atividades deste segmento, com base no método de Custos $\mathrm{ABC}$, emergindo a seguinte questão de pesquisa: Como formar preço de venda na prestação de serviços de análises físico-químicas para estudos ambientais utilizando o método de Custeio Baseado em Atividades - ABC? O objetivo geral é apresentar os resultados de um estudo de caso que analisou a utilização do custeio $\mathrm{ABC}$ para a formação de preço de venda na prestação de serviços de um laboratório de análises físico-químicas para estudos ambientais. Para atingir o objetivo geral têm-se os seguintes objetivos específicos: i) identificar os tipos de serviços prestados e as atividades requeridas; ii) identificar os recursos consumidos, os direcionadores de recursos e de atividades; iii) apurar o custo do serviço prestado; e iv) formar o preço de venda com base no mark up e compará-los com praticados pela entidade.

O laboratório locus da pesquisa localiza-se no sul do Estado de Santa Catarina e pertence a uma Instituição de Ensino Superior (IES) que entre suas atividades presta serviços de análises físico-químicas, no qual foram identificados os serviços prestados, as atividades necessárias para realizar estes serviços, bem como o consumo de recursos para desenvolver essas atividades, de modo a identificar os custos por serviço ofertado. Destaca-se que os serviços prestados pelo laboratório são essenciais para consolidar a responsabilidade socioambiental das empresas no que se refere ao cumprimento das obrigações legais inerentes as legislações ambientas e auxiliar os órgãos públicos fiscalizadores na execução dessas atividades.

Os resultados desse estudo poderão ser utilizados como instrumento nos diversos níveis gerenciais da instituição pesquisada, contribuindo na definição de uma estratégia mais adequada de formação de preço de venda relacionado ao setor de prestação de serviços. Além disso, essa pesquisa contribui com os estudos pertinentes ao método de custeio ABC, pois verificou-se que no âmbito nacional não se encontram estudos específicos sobre esse método de custeio em laboratórios de análises físico-químicas para estudos ambientais.

No entanto, estudos como Ribeiro e Camacho (2006) que abordou a utilização do Custeio Baseado em Atividades (ABC) em um CMEI - Centro Municipal de Educação Infantil apontou esse método de custeio como eficiente e adequado. Com sua aplicação foi possível apurar o custo do serviço prestado por turma, identificar o custo por aluno e visualizar a forma como os recursos foram consumidos pelas atividades, bem como o custo dessas atividades.

Torres, Silva e Matsumoto (2006) buscaram demonstrar que pelo método de custeio $\mathrm{ABC}$, os produtos são custeados pelos valores efetivamente gastos para produzi-los, sejam gastos diretos ou indiretos. Ademais é possível aplicar esse método também nas empresas prestadoras de serviços, não apenas às indústrias, pois permite melhor visualização dos custos, devido à análise das atividades executadas.

Cavalcanti (2011) apresentou estudo de caso de aplicação do método de custeio ABC em uma organização prestadora de serviços de saúde. A pesquisa possibilitou identificar como os recursos são consumidos na prestação dos serviços, mensurar a lucratividade dos serviços prestados e desenvolver política de controle de custos adequada. Foram identificadas atividades que não agregam valor, as quais poderiam ser eliminadas, reduzindo os custos indiretos.

A pesquisa de Borinelli e dos Santos (2014) teve a finalidade de verificar se a implantação do método $\mathrm{ABC}$ impacta o nível de confiabilidade das informações em uma Instituição Financeira. Os resultados indicaram que as informações geradas pelo $\mathrm{ABC}$ aumentam o grau de confiabilidade, permitindo maior segurança no controle e nas tomadas de decisões na percepção dos gestores.

Assim, a realização desse tipo de pesquisa se justifica, uma vez que laboratórios de análises físico-químicas para estudos ambientais têm papel fundamental, por fornecer subsídios para as empresas e órgãos fiscalizadores da região em que estão inseridos para o cumprimento de obrigações legais, definidas pelas legislações ambientais vigente no âmbito federal, estadual e municipal. Desse modo, contribuem com a formação de uma sociedade sustentável. As 
entidades que atuam na prestação desse tipo de serviços geram emprego e renda, além de cooperar com o desenvolvimento social e econômico do país.

Esse artigo está organizado em cinco seções: (1) introdução; (2) conceitos sobre o Custeio ABC e formação do preço de venda; (3) procedimentos metodológicos; (4) análises e discussão dos resultados; e (5) considerações finais.

\section{Custeio ABC e Formação do Preço de Venda}

$\mathrm{O}$ custeio $\mathrm{ABC}$ teve origem com o objetivo de solucionar as incertezas geradas por rateios arbitrários utilizados para distribuição dos custos indiretos pelos métodos mais tradicionais de custeio, como o custeio por absorção, por exemplo. O ABC difere-se dos demais métodos pelo fato de tratar os custos indiretos de forma mais específica, monitorando o fato gerador do custo indireto e direcionando ao produto ou serviços. Assim, torna a relação produto e custo mais fidedignos, evita os possíveis equívocos gerados pelos métodos que têm como princípio o rateio dos custos indiretos (FERREIRA, 2016).

$\mathrm{O}$ ABC é um instrumento de gestão relevante que fornece aos seus usuários a possibilidade de identificar possíveis gastos desnecessários nas atividades de uma entidade, compreendendo a forma que estas estão relacionadas com a geração de receita e o consumo de recursos (SANTOS et al., 2015). Ademais, esse método apresenta os custos dos produtos de uma maneira mais lógica, diferente dos demais métodos, garantindo que não sejam distorcidos por rateios tantas vezes arbitrários (MARTINS, 2003).

O sistema de Custeio Baseado em Atividades, segundo Martins (2003), apresenta dois grandes enfoques: a) contribui com o aperfeiçoamento do processo produtivo visando a redução dos custos e identifica gastos que não contribuem de forma efetiva na elaboração do produto ou serviço prestado; b) na formação do preço de venda, avaliando os custos do processo produtivo e relaciona-os ao preço de venda, na busca de valor final do produto mais competitivo no mercado.

Além disso, é possível identificar os custos pelo método $\mathrm{ABC}$ e compará-los com padrões pré-fixados gerando resultados positivos ou negativos, com a finalidade de controlar os gastos dos departamentos e observar se estes estão de acordo com o que foi planejado (DUBOIS; KULPA; SOUZA, 2006).

Para compreender como funciona o $\mathrm{ABC}$ é necessário o entendimento de algumas definições básicas utilizadas e suas aplicações nesse método de custeio. Os recursos são os bens e serviços que as atividades consomem, tais como: mão de obra, aluguéis, seguros, materiais de expediente, entre outros (MARTINS, 2003). Entende-se que os recursos são consumidos pelas atividades, que por sua vez são absorvidas pelos produtos ou serviços (FERREIRA, 2016).

As atividades são as ações necessárias para realizar a elaboração e comercialização de um determinado produto ou prestação de serviços. Para Santos (2011), as atividades podem ser definidas como conjunto de tarefas e atos relacionados a um sistema organizacional, que são necessárias para desenvolvimento dos serviços prestados pela empresa.

A identificação dos direcionadores de custos é o principal fator do sucesso do sistema ABC. Pode-se definir como direcionadores a causa e efeito relacionado com consumo de recursos por uma atividade ou utilização desta por um produto. Sendo este o principal fator determinante, pois indicará o fato gerador do gasto que irá custear a atividade ou como uma atividade será consumida por um produto (DUBOIS; KULPA; SOUZA, 2006).

No que se refere aos direcionadores deve-se distinguir os dois tipos existentes. $\mathrm{O}$ primeiro direcionador é o de custos, que define o quanto uma atividade consome de um recurso. O segundo é o direcionador de atividade, que define a maneira como uma atividade é utilizada por um produto o serviço prestado (MARTINS, 2003). 
De modo prático, Martins (2003) afirma que, para implantação do custeio ABC, é necessário percorrer as seguintes etapas: coleta das informações; descrição das atividades; descrição dos recursos; definição dos direcionadores de recursos; direcionar recursos; definir direcionadores de atividades; alocar atividades e analisar os resultados obtidos.

Para Torres, Silva e Matsumoto (2006), atualmente as organizações buscam alternativas para reduzir os custos de seus produtos e eliminar os desperdícios de modo a apresentar preço de venda mais competitivo. O método de custeio $\mathrm{ABC}$ surgiu para contribuir com esse processo, uma vez que trata os custos indiretos de maneira diferenciada contribui para evitar distorções arbitrárias de rateio, devido à apropriação dos recursos às atividades e posteriormente a locação dos custos das atividades aos produtos e serviços. Assim, tem se demostrado ser uma excelente ferramenta para empresas que tem por características apresentar valores relevantes de custos indiretos em seu processo de produção de bens e serviços (FERREIRA, 2016; MARTINS, 2003).

Em relação à formação do preço de venda, este pode ser determinado com base nos custos, no consumidor ou na concorrência. No método baseado no consumidor as empresas consideram a percepção dos consumidores sobre o valor do produto, ou seja, o quanto estão dispostos a pagar por ele. O método baseado na concorrência determina que os preços dos produtos e serviços sejam atribuídos conforme os preços praticados pelos seus concorrentes. No método baseado no custo, deve-se aplicar uma margem, denominada mark-up, necessária para cobrir os gastos que não estão inseridos nos custos como os tributos, comissões e lucro desejado. No método misto, o preço de venda poderá contribuir para a maximização dos lucros, atender ao mercado consumidor e melhorar os níveis de produção da entidade (BRUNI, FAMÁ, 2004; DUBOIS, KULPA, SOUZA, 2006; MARTINS, 2003; MEGLIORINI, 2007).

\section{Procedimentos Metodológicos}

A pesquisa é descritiva, pois apresenta os serviços prestados no ramo de laboratórios de análises físico-químicas para estudos ambientais; os gastos para realização destes serviços; e uma proposta para formação do preço de venda com base no custeio ABC. O principal objetivo da pesquisa descritiva é identificar e registrar os acontecimentos, para que possam ser analisados e qualificados, mas com a certeza que as informações não sofrerão interferência pelo pesquisador (ANDRADE, 2007).

A abordagem do problema tem caráter qualitativo. Segundo Diehl (2004), tal estudo pode descrever características de um determinado grupo social, com objetivo de apresentar seu comportamento e, assim, promover um melhor entendimento de determinado grupo.

A pesquisa é documental com base em dados fornecidos pela organização. Conforme Gil (1996), a pesquisa documental é similar à pesquisa bibliográfica, tendo como principal diferença a origem dos dados a serem analisados. Caracteriza-se também como estudo de caso, uma vez que esse permite, segundo Yin (2010), a compreensão dos fatos reais inseridos no cotidiano de uma sociedade abrangendo situações que possam ser integradas ao contexto histórico.

O estudo de caso foi realizado em um laboratório de análises físico-químicas para estudos ambientais, pertencente a um instituto de pesquisa de uma IES (Instituição de Ensino Superior), localizado no sul de Santa Catarina, que tem como principal atividade a educação superior. O laboratório contribui de forma relevante para assegurar a sustentabilidade financeira da entidade, bem como para o desenvolvimento regional. O laboratório presta 12 tipos de serviços que estão relacionados a duas diferentes matrizes: a) Matriz 1 - análises de emissões atmosféricas em dutos e chaminés; e b) Matriz 2 - monitoramento da qualidade do ar em nível do solo. 
$\mathrm{Na}$ pesquisa documental foram analisadas as seguintes informações gerenciais: i) demonstrativos de resultado do ano de 2016; ii) inventários de imobilizados; e iii) estudos relacionados com as demandas e horas técnicas dos serviços prestados realizados no ano de 2015. A coleta de dados ocorreu no período de março a maio de 2017, por um dos autores do trabalho, caracterizando a pesquisa como participante, pois o indivíduo trabalha no local pesquisado (PRODANOV; FREITAS, 2013). Também, foi realizada entrevista com o responsável técnico do laboratório.

Inicialmente foram identificados os serviços prestados, as atividades necessárias para realizar estes serviços, bem como o consumo de recursos para desenvolver essas atividades, de modo a possibilitar a distribuição dos custos aos serviços prestados. Esses dados foram tabulados em planilhas eletrônicas da Microsoft Excel e, após definidas as premissas para taxa de Mark-up, foi calculado o preço de venda, comparando-os com os praticados pela empresa.

\section{Descrição e Análise dos Resultados}

Nessa seção expõe-se a descrição e análises dos dados no intuito de formação do preço de venda com base na aplicação do custeio ABC.

\subsection{Aplicação do método ABC}

Para a aplicação do método de custeio $\mathrm{ABC}$ foram realizadas as seguintes etapas: a) descrição das atividades; b) identificação dos recursos e definição dos seus direcionadores; c) alocação dos recursos nas respectivas atividades; d) alocação dos custos diretos nas atividades; e) definição dos direcionadores de atividades; e por fim, f) composição dos custos aos serviços prestados.

O Quadro 1 apresenta a descrição dos grupos de atividades subdividido em atividades e tarefas realizadas no laboratório.

Quadro 1 - Descrição das atividades

\begin{tabular}{|c|c|c|}
\hline $\begin{array}{l}\text { Grupos de } \\
\text { Atividades }\end{array}$ & Atividades & Tarefas \\
\hline \multirow{5}{*}{$\begin{array}{l}1 \text { Comercial e } \\
\text { Gerencial - CML }\end{array}$} & \multirow{5}{*}{ 1.1. Elaborar proposta comercial } & Contato com Cliente \\
\hline & & Cadastrar cliente \\
\hline & & Elaborar orçamento \\
\hline & & Efetivação no sistema \\
\hline & & Formalizar contrato \\
\hline \multirow{7}{*}{$\begin{array}{l}2 \text { Amostragem de } \\
\text { Campo - AMC }\end{array}$} & \multirow{4}{*}{ 2.1. Planejar as amostragens } & Agendar amostragem \\
\hline & & $\begin{array}{l}\text { Definir equipe de amostragem } \\
\text { Processar informações de coleta }\end{array}$ \\
\hline & & Preparar material de amostragens \\
\hline & & Definição dos pontos de amostragem \\
\hline & $\begin{array}{l}\text { 2.2. Realizar amostragem de Emissões } \\
\text { atmosféricas }\end{array}$ & Operação do equipamento \\
\hline & 2.3. Realizar avaliação de ruído & Operar equipamento \\
\hline & 2.4. Realizar amostragem da Qualidade do AR & Operar do equipamento \\
\hline $\begin{array}{l}3 \text { Análises } \\
\text { Laboratoriais - ANA }\end{array}$ & 3.1. Preparar amostra & $\begin{array}{l}\text { Preparar amostra para análise } \\
\text { Processar resultados de análises }\end{array}$ \\
\hline
\end{tabular}




\begin{tabular}{|l|l|l|}
\hline \multirow{1}{*}{} & $\begin{array}{l}\text { 3.2. Realizar Análises de CO, CO2 e NOX de } \\
\text { Emissões Atmosféricas }\end{array}$ & Operação do equipamento \\
\cline { 2 - 3 } & $\begin{array}{l}\text { 3.3. Realizar análise de Material Particulado } \\
\text { em chaminé e na qualidade do ar. }\end{array}$ & Analisar Amostra \\
\cline { 2 - 3 } & $\begin{array}{l}\text { 3.4 Realizar Análises Realizar Análises de } \\
\text { SOX (SO2, SO3 e Nevoas de H2SO4) }\end{array}$ & Analisar Amostra \\
\cline { 2 - 3 } & 3.5. Realizar Análise de NO2 & Analisar Amostra \\
\cline { 2 - 3 } & 3.6. Realizar análise de Fumaça & Analisar Amostra \\
\hline \multirow{2}{*}{ Relatórios - REL } & \multirow{2}{*}{ 4.1. Emitir relatório } & Elaborar relatórios resultado \\
\cline { 2 - 3 } & & Enviar relatório cliente \\
\cline { 2 - 3 } & Efetivar faturamento \\
\hline
\end{tabular}

Fonte: Dados da pesquisa (2017).

Os grupos de atividades necessárias para a prestação dos serviços em estudo são: 1) comercial e gerencial (CML); 2) amostragem de campo (AMC); 3) análises laboratoriais (ANA); e 4) Relatórios (REL); as quais foram subdivididas em 12 atividades codificadas.

Para desenvolvimento do estudo proposto foram identificados os recursos com base no Demonstrativo de Resultado (DR) do laboratório. Assim, com uma análise do processo da prestação de serviço definiu-se os direcionadores dos recursos às atividades.

O Quadro 2 apresenta os recursos e os direcionadores definidos inicialmente para os grupos de atividades, que posteriormente foram utilizados para alocação dos custos às atividades.

Quadro 2 - Recursos e seus direcionadores

\begin{tabular}{|c|c|c|c|c|c|}
\hline \multirow{2}{*}{ Recursos } & \multirow{2}{*}{ Direcionadores } & \multicolumn{4}{|l|}{ Função } \\
\hline & & 1-CML & 2-AMC & 3-ANA & 4-REL \\
\hline \multicolumn{6}{|c|}{ Gastos Indiretos } \\
\hline Salários, encargos e benefícios & Tempo (hora técnica) & $\mathrm{x}$ & $\mathrm{x}$ & $\mathrm{x}$ & $\mathrm{x}$ \\
\hline Energia & Utilização de área - m² & $\mathrm{x}$ & $\mathrm{x}$ & $\mathrm{x}$ & $\mathrm{x}$ \\
\hline Depreciação equipamentos & Tempo de consumo & & $\mathrm{x}$ & $\mathrm{x}$ & \\
\hline Manutenção equipamento & Tempo de consumo & & $\mathrm{x}$ & $\mathrm{x}$ & \\
\hline Calibração equipamento & Tempo de consumo & & $\mathrm{x}$ & $\mathrm{x}$ & \\
\hline Material de limpeza & Utilização de área - m² & $\mathrm{x}$ & $\mathrm{x}$ & $\mathrm{x}$ & $\mathrm{x}$ \\
\hline Segurança do trabalho & Quantidade de técnicos & & $\mathrm{x}$ & $\mathrm{x}$ & \\
\hline Uniforme & Quantidade de técnicos & & $\mathrm{x}$ & $\mathrm{x}$ & \\
\hline Depreciação de computadores & Número de computadores & $\mathrm{x}$ & $\mathrm{x}$ & $\mathrm{x}$ & $\mathrm{x}$ \\
\hline Taxas e impostos do Imóvel & Utilização de área - m² & $\mathrm{x}$ & $\mathrm{x}$ & $\mathrm{x}$ & $\mathrm{x}$ \\
\hline Depreciação de móveis e utensílio & Quantidade utilizada & $\mathrm{x}$ & $\mathrm{x}$ & $\mathrm{x}$ & $\mathrm{x}$ \\
\hline \multicolumn{6}{|c|}{ Gastos Diretos } \\
\hline Depreciação de veículo & Direto & & $\mathrm{x}$ & & \\
\hline Manutenção de veículo & Direto & & $\mathrm{x}$ & & \\
\hline Prêmios de seguros veículo & Direto & & $\mathrm{x}$ & & \\
\hline Combustível & Direto & & $\mathrm{x}$ & & \\
\hline Tributos do veículo & Direto & & $\mathrm{x}$ & & \\
\hline Telefone & Direto & $\mathrm{x}$ & & & \\
\hline Fotocópias & Direto & & & & $\mathrm{x}$ \\
\hline Correio & Direto & & & & $\mathrm{x}$ \\
\hline
\end{tabular}




\begin{tabular}{|l|l|l|l|l|l|}
\hline Serviço terceirizado & Direto & & & $\mathrm{x}$ & \\
\hline Insumos & Direto & & & $\mathrm{x}$ & \\
\hline Viagens e estadas & Direto & & $\mathrm{x}$ & & \\
\hline
\end{tabular}

Fonte: Dados da pesquisa (2017).

Em seguida, apresentam-se os gastos com mão de obra, seguido pelos gastos com equipamentos, demais gastos indiretos e diretos e totais por atividades.

O laboratório possui seis funcionários distribuídos conforme Tabela 1.

Tabela 1 - Recurso Mão de Obra Cargos $(\mathrm{R} \$)$

\begin{tabular}{|c|c|c|c|c|c|c|c|}
\hline 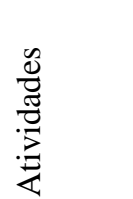 & 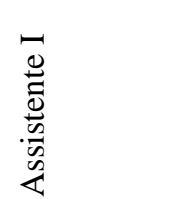 & 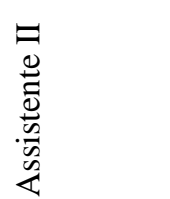 & 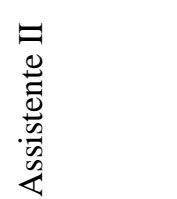 & 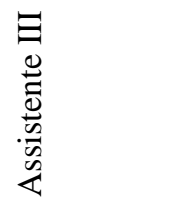 & 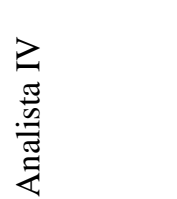 & 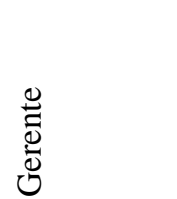 & $\stackrel{\vec{J}}{0}$ \\
\hline 1.1 & & & & $17.990,78$ & $3.513,83$ & $19.181,75$ & $40.686,36$ \\
\hline 2.1 & $28.222,79$ & $26.169,32$ & $32.757,00$ & & $20.520,79$ & & $107.669,90$ \\
\hline 2.2 & $11.519,51$ & $11.564,08$ & $4.635,42$ & & $1.054,15$ & & $28.773,16$ \\
\hline 2.3 & $1.950,16$ & & $2.092,64$ & & 475,89 & & $4.518,69$ \\
\hline 2.4 & & $1.861,85$ & $1.890,67$ & & 429,96 & & $4.182,48$ \\
\hline 3.1 & $6.623,72$ & $11.868,40$ & $10.506,96$ & & $3.724,66$ & & $32.723,74$ \\
\hline 3.2 & 431,98 & & 463,54 & & 105,41 & & $1.000,93$ \\
\hline 3.3 & $2.835,04$ & $2.995,80$ & $3.042,17$ & & 702,77 & & $9.575,78$ \\
\hline 3.4 & 118,44 & 125,15 & 127,09 & & 28,9 & & 399,58 \\
\hline 3.5 & 138,87 & 146,75 & 149,02 & & 33,89 & & 468,53 \\
\hline 3.6 & 138,87 & 146,75 & 149,02 & & 33,89 & & 468,53 \\
\hline 4.1 & & & & & $66.603,29$ & & $66.603,29$ \\
\hline TOTAL & $51.979,37$ & $54.878,11$ & $55.813,54$ & $17.990,78$ & $97.227,43$ & $19.181,75$ & $297.070,98$ \\
\hline
\end{tabular}

Fonte: Dados da pesquisa (2017).

O gasto com mão de obra (MOD) é composto por salário, encargos e benefícios. O tempo relacionado às atividades foi identificado no estudo de horas realizado pela IES. Assim, a soma de todos os gastos divididos pela carga horária efetiva de cada técnico resultou no custo da MOD por hora, que posteriormente foi multiplicado pelo tempo dispendido em cada atividade. Definiu-se com hora efetiva apenas o tempo do técnico empregado nas funções, desconsiderando sazonalidade do processo.

Observa-se que o gasto com o assistente III, que presta serviços para outros laboratórios da IES, foi alocado pelo tempo realizado na atividade comercial deste laboratório assim como o valor da mão de obra do gerente. Os valores correspondentes aos assistentes I, 
IIa e IIb foram alocados às atividades de acordo com as funções de amostragem e análise conforme o tempo. O valor do Analista IV foi direcionado principalmente a atividade do relatório, mas também são consumidas suas horas em todo o processo, pois este é responsável pela análise técnica dos orçamentos e supervisionar as atividades de laboratório e amostragem.

A taxa de depreciação foi definida conforme tempo de vida útil, informadas pelo responsável técnico do laboratório, baseado em dados históricos e referências do fabricante. A Tabela 02 apresenta os recursos consumidos com depreciação, acrescidos pela manutenção e calibração para equipamentos alocados nas suas respectivas atividades.

Tabela 2 - Depreciação, manutenção e calibração com equipamentos

Atividade Código

Equipamentos

1.1. Elaborar proposta comercial

2.1. Planejar as amostragens

Continuação.

2.2. Realizar amostragem de Emissões atmosféricas

2.3. Realizar avaliação de ruído

2.4. Realizar amostragem da Qualidade do AR

3.1. Preparar amostra

3.2 Realizar Análises de CO, CO2 e NOX de Emissões Atmosféricas

3.3. Realizar análise de Material Particulado em chaminé e na qualidade do ar.

3.4 Realizar Análises Realizar Análises de SOX (SO2, SO3 e Nevoas de H2SO4)

3.5 Realizar Análise de NO2

3.6. Realizar análise de Fumaça

4.1. Emitir relatório

Total

Fonte: Dados da pesquisa (2017).
8

$\mathrm{R} \$ 249,00$

$6,8,9$ e 11

$\mathrm{R} \$ 583,90$

1

$\mathrm{R} \$ 11.831,53$

7

$\mathrm{R} \$ 107,99$

2,3 e 4

$\mathrm{R} \$ 18.035,00$

8

$\mathrm{R} \$ 166,00$

5

$\mathrm{R} \$ 1.200,00$

$6,8,9$ e 11

$\mathrm{R} \$ 334,90$

6

$\mathrm{R} \$ 59,45$

10

$\mathrm{R} \$ 250,00$

0

9

$\mathrm{R} \$ 264,00$

$\mathrm{R} \$ 33.081,77$

Observa-se que as atividades que apresentaram maior consumo relacionado aos recursos com equipamentos foram: 2.2 Realizar amostragem de emissões atmosféricas, na razão de 35,76 $\%(\mathrm{R} \$ 11.831,53)$ do total $(\mathrm{R} \$ 33.081,77)$; e atividade 2.4 Realizar amostragem da qualidade do $\mathrm{AR}$, que consomiu 54,53\% (R\$ 18.035,00) desses gastos, pois utilizam equipamentos com valores mais representativos. A avaliação de ruído consome integralmente o custo com o equipamento decibelímetro, na atividade 3.6 não houve gastos com equipamentos, pois essa parte do processo o laboratório terceiriza. 
Os demais gastos indiretos foram alocados às atividades conforme direcionadores definidos no Quadro 2. Logo, na Tabela 3 são expostos os totais dos demais gastos indiretos.

Tabela 3 - Demais gastos indiretos

\begin{tabular}{|c|c|c|c|c|c|c|c|c|}
\hline 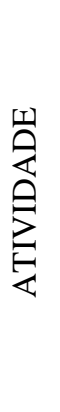 & 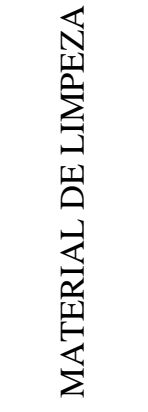 & 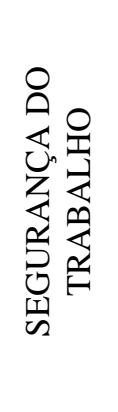 & 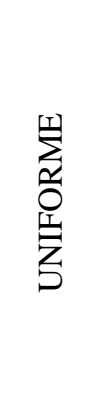 & 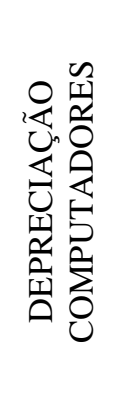 & 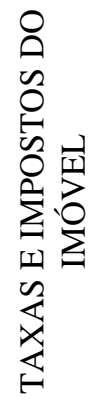 & 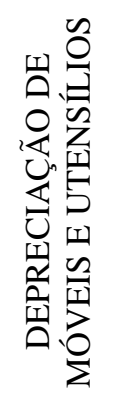 & $\begin{array}{l}\mathbb{J} \\
\frac{1}{\square} \\
\underset{I}{\mid}\end{array}$ & 它 \\
\hline 1.1 & $1.856,10$ & & & 312,93 & 29,70 & 121,80 & 198,00 & $2.518,17$ \\
\hline 2.1 & $1.237,40$ & & & 156,47 & 19,80 & 81,20 & 132,00 & $1.626,68$ \\
\hline 2.2 & & 281,87 & 94,67 & 156,47 & & & & 532,82 \\
\hline 2.3 & & 281,87 & 94,67 & & & & & 376,54 \\
\hline 2.4 & & 281,87 & 94,67 & & & & & 376,54 \\
\hline 3.1 & $1.237,40$ & & & 156,47 & 19,80 & 81,20 & 132,00 & $1.626,68$ \\
\hline 3.3 & $2.474,80$ & 70,47 & & & 39,60 & 162,40 & 264,00 & $3.011,27$ \\
\hline 3.4 & $1.856,10$ & 70,47 & & & 29,70 & 121,80 & 198,00 & $2.276,07$ \\
\hline 3.5 & $1.237,40$ & 70,47 & & & 19,80 & 81,20 & 132,00 & $1.540,87$ \\
\hline 4.1 & $2.474,80$ & 352,33 & & 156,47 & 39,60 & 162,40 & 264,00 & $3.449,41$ \\
\hline
\end{tabular}

Fonte: Dados da pesquisa (2017).

Nota-se que os gastos relacionados à limpeza, energia e móveis utensílios foram alocados nas atividades que utilizam o espaço físico da IES. As atividades 2.1 Planejar as amostragens, 2.2 . Realizar amostragem de Emissões atmosféricas, 3.1 Preparar amostra e 4.1 Emitir relatório possuem um computador cada, exceto atividade 1.1 Elaborar proposta comercial que possui 2 computadores, sendo assim o gasto com depreciação dos computadores foi mais expressivo para atividade comercial e gerencial. Os uniformes são utilizados pelos técnicos que realizam as atividades de amostragem; os recursos com segurança do trabalho estão relacionados com os trabalhos de amostragem, laboratoriais e relatório.

A Tabela 4 apresenta o total dos gastos indiretos alocados às atividades.

Tabela 4 - Total dos gastos indiretos

\begin{tabular}{lrrrr}
\hline Atividade & Mão de obra & Equipamentos & Demais gastos & Total \\
\hline 1.1 & $40.686,36$ & $\mathrm{R} \$ 249,00$ & 2518,17 & $43.453,53$ \\
2.1 & $107.669,90$ & $\mathrm{R} \$ 583,90$ & 1626,68 & $109.880,48$ \\
2.2 & $28.773,16$ & $11.831,53$ & 532,82 & $41.137,51$ \\
2.3 & $4.518,69$ & $\mathrm{R} \$ 107,99$ & 376,54 & $5.003,22$ \\
2.4 & $4.182,48$ & $18.035,00$ & 376,54 & $22.594,02$ \\
3.1 & $32.723,74$ & 166,00 & 1626,68 & $34.516,42$ \\
3.2 & $1.000,93$ & $1.200,00$ & 0,00 & $2.200,93$ \\
3.3 & $9.575,78$ & 334,90 & 3011,27 & $12.921,95$ \\
3.4 & 399,58 & 59,45 & 2276,07 & $2.735,10$ \\
3.5 & 468,53 & 250,00 & 1540,87 & $2.259,40$ \\
3.6 & 468,53 & 0,00 & 0,00 & 468,53
\end{tabular}




\begin{tabular}{lrrrr}
4.1 & $66.603,29$ & 264,00 & 3449,41 & $70.316,70$ \\
Total & $297.070,97$ & $33.081,77$ & $17.335,06$ & $347.487,80$ \\
\hline
\end{tabular}

Fonte: Dados da pesquisa (2017).

Observa-se que para prestação de serviços os gastos relacionados à mão de obra $(\mathrm{R} \$$ 297.070,97) são os mais expressivos, correspondendo a aproximadamente $85 \%$ dos gastos indiretos totais $(\mathrm{R} \$ 347.487,80)$. Os gastos com equipamentos ( $\mathrm{R} \$ 33.081,77)$ equivalem a $10 \%$ e os demais gastos $(17.335,06) 5 \%$ dos custos indiretos totais.

Em seguida, foram identificados os gastos diretos das atividades, que dispensam a necessidade do uso de direcionadores de recursos. A Tabela 5 apresenta os gastos diretos por atividade que são: telefone, fotocópias, correio, serviços de terceiros e materiais diversos.

Tabela 5 - Gastos Diretos

\begin{tabular}{|c|c|c|c|c|c|c|c|c|c|c|}
\hline \multirow[b]{2}{*}{ 离 } & \multicolumn{10}{|c|}{ Recurso (R\$) } \\
\hline & 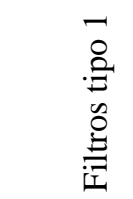 & 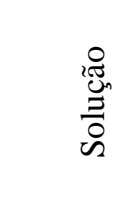 & $\frac{\frac{\tilde{D}}{0}}{\frac{\vec{d}}{\omega}}$ & $\begin{array}{l}\frac{\pi}{0} \\
\frac{0}{0} \\
\frac{0}{4}\end{array}$ & 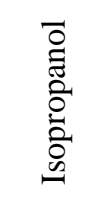 & 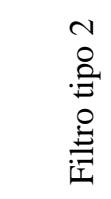 & & 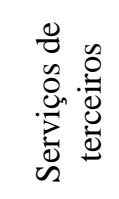 & 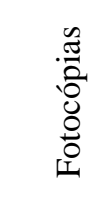 & 氶 \\
\hline 1.1 & & & 32 & & & & & & & 32 \\
\hline 3.3 & 5.467 & & & 900 & & & & & & 6.367 \\
\hline 3.4 & & & & & 900 & & & & & 900 \\
\hline 3.5 & & 200 & & & & & & & & 200 \\
\hline 3.6 & & & & & & 100 & & 1200 & & 1300 \\
\hline 4 & & & & & & & 500 & & 812 & 1312 \\
\hline Total & 5.467 & 200 & 32 & 900 & 900 & 100 & 500 & 1200 & 812 & 10.111 \\
\hline
\end{tabular}

Fonte: Dados da pesquisa (2017).

Os recursos diretos foram distribuídos da seguinte forma: 1 - fotocópia e correio estão relacionados diretamente com a elaboração da atividade de relatórios; 2 - filtro tipo 1 e acetona estão relacionados diretamente com análise de material particulado na qualidade do ar e emissões atmosféricas em dutos e chaminés; 3 - custos com isopropanol está alocado na análise de SOX; 4 - filtro tipo 2 e serviços de terceiros estão relacionados a análise de fumaça; 5 soluções para análise de NO2; e telefone está relacionado a atividade comercial e gerencial.

Após o reconhecimento e direcionamento dos recursos diretos e indiretos foi possível identificar gastos totais relacionados às atividades que são apresentados na Tabela 6.

Tabela 6 - Total de gastos por atividade

Atividades $\quad$ Gasto indiretos $(\mathrm{R} \$) \quad$ Gasto diretos $(\mathrm{R} \$) \quad$ Total dos gastos $(\mathrm{R} \$) \quad$ Análise vertical

\begin{tabular}{lrrrr}
\hline 1.1 & $43.453,53$ & 32,00 & $43.485,53$ & $12 \%$ \\
2.1 & $109.880,48$ & - & $109.880,48$ & $31 \%$ \\
2.2 & $41.137,51$ & - & $41.137,51$ & $12 \%$
\end{tabular}




\begin{tabular}{lrrrr}
2.3 & $5.003,22$ & - & $5.003,22$ & $1 \%$ \\
2.4 & $22.594,02$ & - & $22.594,02$ & $6 \%$ \\
3.1 & $34.516,42$ & - & $34.516,42$ & $10 \%$ \\
3.2 & $2.200,93$ & - & $2.200,93$ & $1 \%$ \\
3.3 & $12.921,95$ & $6.367,00$ & $19.288,95$ & $5 \%$ \\
3.4 & $2.735,10$ & 900,00 & $3.635,10$ & $1 \%$ \\
3.5 & $2.259,40$ & 200,00 & $2.459,40$ & $1 \%$ \\
3.6 & 468,53 & $1.300,00$ & $1.768,53$ & $0 \%$ \\
4.1 & $70.316,70$ & $1.312,00$ & $71.628,70$ & $20 \%$ \\
Total $(\mathrm{R} \$)$ & $347.487,80$ & $10.111,00$ & $357.598,80$ & $100 \%$ \\
\hline
\end{tabular}

Fonte: Dados da pesquisa (2017).

Verifica-se que atividade 2.1 planejamento de amostragem e atividade 4.1 emissão de relatório são as que mais consomem recursos correspondendo a razão de $31 \%$ ( $\mathrm{R} \$ 109.880,48$ ) e $20 \%$ ( $\mathrm{R} \$ 71.628,70)$, respectivamente. Observa-se que os gastos indiretos direcionados as atividades, correspondem a $97 \%$ de todos os recursos.

Para cálculo do custo de cada serviço, foram definidos os direcionadores das atividades e posteriormente alocados nos serviços prestados. O direcionador definido para atividade 1.1 está relacionado aos orçamentos aprovados para cada serviço prestado. Para as atividades 2.1, 2.4, 3.1, 3.3, 3.4, 3.5, 3.6, 4,1, foram definidos os direcionadores com base no volume de amostra. Ressalta-se que para elaboração de relatório é mais representativo trabalhar com número de amostras do que por relatório produzido, pois muitos relatórios elaborados possuem mais de uma amostra.

A atividade 3.1 é realizada diretamente na empresa com medições em tempo real pelo equipamento, sendo possível direcionar pela quantidade de análise realizada. As atividades 2.2 e 2.3 estão relacionadas diretamente aos seus respectivos serviços.

Para maior entendimento do funcionamento do processo produtivo do laboratório, o Quadro 3 apresenta a sistemática de como as atividades são consumidas pelos serviços prestados e os gastos atrelados a cada serviço.

Quadro 3 - Alocação das atividades nos serviços e custo dos serviços baseado no ABC

\begin{tabular}{|c|c|c|c|c|c|c|c|c|c|c|c|c|c|c|c|c|}
\hline$\stackrel{\bigcirc}{\varrho}$ & Serviços & $\stackrel{\nabla}{\sim}$ & $\vec{\sim}$ & $\underset{\sim}{N}$ & $\stackrel{n}{\sim}$ & $\stackrel{\nabla}{\sim}$ & $\vec{m}$ & $\stackrel{\sim}{m}$ & $\ddot{m}$ & $\stackrel{\nabla}{\dot{r}}$ & $n$ & $\stackrel{\varphi}{\dot{m}}$ & $\vec{\forall}$ & $\begin{array}{c}\text { Gastos } \\
\text { Totais } \\
(\mathrm{R} \$)\end{array}$ & $\mathrm{N}^{\mathrm{o}}$ & $\begin{array}{c}\text { Gasto } \\
\text { Unitário } \\
(\mathrm{R} \$)\end{array}$ \\
\hline \multirow{5}{*}{ 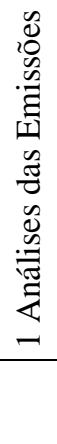 } & $\begin{array}{l}\text { 1.1 Amostragem de } \\
\text { dutos e chaminés }\end{array}$ & $X$ & $\mathrm{X}$ & $X$ & & & $X$ & $\mathrm{X}$ & & & & & $X$ & $65.137,31$ & 90 & 585,19 \\
\hline & $\begin{array}{l}\text { 1.2 Determinação } \\
\text { de CO }\end{array}$ & $X$ & $X$ & & & & $X$ & $\mathrm{X}$ & & & & & $X$ & $22.666,47$ & 85 & 259,57 \\
\hline & $\begin{array}{l}\text { 1.3 Determinação } \\
\text { de MP }\end{array}$ & $X$ & $X$ & & & & $X$ & & $\mathrm{X}$ & & & & $X$ & $26.526,50$ & 90 & 292,82 \\
\hline & $\begin{array}{l}\text { 1.4 Determinação } \\
\text { de NO2 }\end{array}$ & $X$ & $X$ & & & & $X$ & $X$ & & & & & $X$ & $7.999,93$ & 30 & 259,57 \\
\hline & $\begin{array}{l}\text { 1.5 Determinação } \\
\text { de SOX }\end{array}$ & $X$ & $X$ & & & & $X$ & & & $\mathrm{X}$ & & & $X$ & $4.805,17$ & 17 & 280,98 \\
\hline \multirow{3}{*}{ 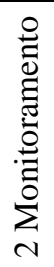 } & $\begin{array}{l}\text { 2.1 Monitoramento } \\
\text { de PTS }\end{array}$ & $X$ & $X$ & & & $X$ & $X$ & & $X$ & & & & $\mathrm{X}$ & $\begin{array}{c}111.965,3 \\
9\end{array}$ & 340 & 299,80 \\
\hline & $\begin{array}{l}\text { 2.2 Monitoramento } \\
\text { de } \mathrm{SO} 2\end{array}$ & $X$ & $X$ & & & $X$ & $X$ & & & $\mathrm{X}$ & & & $X$ & $37.768,18$ & 119 & 287,96 \\
\hline & $\begin{array}{l}\text { 2.3 Monitoramento } \\
\text { de NO2 }\end{array}$ & $X$ & $X$ & & & $X$ & $X$ & & & & $\mathrm{X}$ & & $X$ & $36.153,41$ & 116 & 280,71 \\
\hline
\end{tabular}




\begin{tabular}{|l|l|l|l|l|l|l|l|l|l|l|l|l|l|l|l|c|}
$\begin{array}{l}2.4 \text { Monitoramento } \\
\text { de MP10 }\end{array}$ & $\mathrm{X}$ & $\mathrm{X}$ & & & $\mathrm{X}$ & $\mathrm{X}$ & & $\mathrm{X}$ & & & & $\mathrm{X}$ & $12.857,61$ & 39 & 299,80 \\
\hline $\begin{array}{l}\text { 2.5 Partículas } \\
\text { Sedimentáveis - PS }\end{array}$ & $\mathrm{X}$ & $\mathrm{X}$ & & & & $\mathrm{X}$ & & $\mathrm{X}$ & & & & $\mathrm{X}$ & $8.256,12$ & 28 & 292,82 \\
\hline $\begin{array}{l}\text { 2.6 Monitoramento } \\
\text { de Fumaça }\end{array}$ & $\mathrm{X}$ & $\mathrm{X}$ & & & $\mathrm{X}$ & $\mathrm{X}$ & & & & & $\mathrm{X}$ & $\mathrm{X}$ & $13.082,41$ & 39 & 307,01 \\
\hline $\begin{array}{l}\text { 2.7 Avaliação de } \\
\text { Ruído }\end{array}$ & $\mathrm{X}$ & $\mathrm{X}$ & & $\mathrm{X}$ & & $\mathrm{X}$ & & & & & & $\mathrm{X}$ & $10.380,28$ & 146 & 70,38 \\
\hline
\end{tabular}

Fonte: Dados da pesquisa (2017).

Percebe-se que as atividades 1.1, 2.1, 3.1 e 4.1 foram distribuídas para todos os serviços prestados, $2.3,3.5$ e 3.6 foram alocadas diretamente em seus "produtos". Os serviços que mais consomem atividades foram Amostragem de chaminé ( $\mathrm{R} \$ 65.137,31)$ e determinação de PTS (R\$ $111.965,39$ ), representando $18 \%$ e $31 \%$ respectivamente dos custos totais. O serviço que possui custo unitário mais relevante é a amostragem de chaminé ( $\mathrm{R} \$ 585,19)$, por consumir diretamente os recursos da atividade 2.2.

\subsection{Formação do Preço de Venda}

Para formação do preço de venda baseado no custeio $\mathrm{ABC}$, foi definido o custo unitário do serviço prestado e aplicado o Mark-up multiplicador, baseado no resultado esperado pelos gestores que considerou $10 \%$ como indicador de margem para cobrir as despesas da IES; e 25\% para reinvestimento no próprio laboratório; as quais resultaram em um fator do Mark-up de 1,538462 .

Vale ressaltar que a IES é isenta de impostos, por não possuir finalidades lucrativas, sendo assim, não foi estimado tributos na formação do preço de venda. laboratório.

A Tabela 7 apresenta o preço formado nesse estudo, bem como o praticado pelo

Tabela 7 - Preço de venda praticado X Preço baseado no ABC

\begin{tabular}{lrrr}
\hline Serviços (Relatórios) & Preço praticado(R\$) & Preço ABC(R\$) & Variação \\
\hline M1 - Amostragem de dutos e chaminés & $1.000,00$ & 900,29 & $11 \%$ \\
M1 - Determinação CO & 200,00 & 399,34 & $-50 \%$ \\
M1 - Determinação de MP & 500,00 & 450,49 & $11 \%$ \\
M1 - Determinação de NO2 & 400,00 & 399,34 & $0 \%$ \\
M1 - Determinação de SOX & 500,00 & 432,28 & $16 \%$ \\
M2 - Monitoramento de PTS & 400,00 & 461,23 & $-13 \%$ \\
M2 - Monitoramento de SO2 & 400,00 & 443,02 & $-10 \%$ \\
M2 - Monitoramento de NO2 & 400,00 & 431,86 & $-7 \%$ \\
M2- Monitoramento de MP10 & 400,00 & 461,23 & $-13 \%$ \\
M2 - Partículas sedimentáveis - PS & 150,00 & 450,49 & $-67 \%$ \\
M2 - Monitoramento de Fumaça & 400,00 & 472,32 & $-15 \%$ \\
M2 - Avaliação de ruído & 250,00 & 108,28 & $131 \%$ \\
\hline
\end{tabular}

Fonte: Dados da pesquisa (2017).

Observa-se que para os serviços relacionados a Matriz 1 - análises de emissões atmosféricas em dutos e chaminés, tem-se aplicado preços superiores aos sugeridos pela pesquisa, exceto para análise de determinação de $\mathrm{CO}$ que tem preço abaixo do custo. Contudo, costuma-se realizar venda casada de maior parte dos serviços da Matriz 1, pois estão 
relacionados amostragem de dutos e chaminés, logo não trazem resultados negativos ao laboratório.

Para a Matriz 2 - monitoramento da qualidade do ar em nível do solo, os serviços de maior demanda no período em estudo foram de monitoramento de PTS, SO2 e NO2 e para essas análises o laboratório tem aplicado preços abaixo do sugerido pelo estudo, mas esses preços estão acima do custo mensurado.

Para o serviço de monitoramento de fumaça é importante verificar o custo com terceirização, pois pode afetar o resultado desse serviço, uma vez que o preço praticado se encontra abaixo do sugerido. O monitoramento de PS apresentou preço praticado (R $\$ 150,00$ ) abaixo do custo definido pela pesquisa ( $\mathrm{R}$ \$ 292,82), sendo necessário uma avalição criteriosa na manutenção desse serviço no escopo do laboratório, pois se verificou que este tem baixa demanda.

O serviço que se destacou positivamente foi a avaliação de ruído, sendo aplicado o preço ( $\mathrm{R} \$ 250,00)$ muito acima do calculado com base no custeio ABC. Porém, há politica de aplicação de descontos baseado no volume na contratação de avaliação por monitoramento.

Atualmente o laboratório tem praticado preços com base no mercado. Com a aplicação do método de custeio de $\mathrm{ABC}$ verificou-se os gastos mais relevantes e as atividades com valores mais significativos, o que pode contribuir para controle e redução dos gastos. Contudo, na formação do preço de venda deve ser considerado custo, preço praticado pelo mercado, demanda de cada serviço e o quanto o cliente está disposto a pagar.

\section{Considerações Finais}

O método de custeio $\mathrm{ABC}$ difere-se de outros métodos pela forma de alocar os custos indiretos, ao evitar usar critérios de rateios de forma arbitrária, que possam distorcer o real custo dos produtos e serviços, indicando direcionadores mais lógicos para os recursos consumidos pelas atividades, que serão alocadas posteriormente aos objetos de custeio.

Nesse sentido, esse trabalho objetivou apresentar os resultados de um estudo de caso que analisou a utilização do custeio $\mathrm{ABC}$ para a formação de preço de venda na prestação de serviços de um laboratório de análises físico-químicas para estudos ambientais. O laboratório pertence a uma IES e possui duas matrizes: Matriz 1 - análises de emissões atmosféricas em dutos e chaminés e Matriz 2- monitoramento da qualidade do ar em nível do solo, que contemplam onze tipos de serviços.

Os resultados apontaram que: a) os recursos mais representativos referem-se à mão de obra, equivalente a $85 \%$, seguido dos gastos com equipamentos que correspondem a $10 \%$ dos gastos indiretos totais; b) as atividades que mais consumiram recursos foram 2.1 Planejar as Amostragens e 4.1 Relatórios; c) o serviço que tem custo mais elevado é o Monitoramento de PTS, equivalente ao total de R \$ 111.965,39.

Para formação do preço de venda, pode-se observar que o custo unitário não deve ser o único fator na formação do preço, sendo necessário identificar as demais variáveis como, preço praticado pelo mercado, poder de compra dos clientes e a demanda dos serviços. Assim, a aplicação do método de custeio $\mathrm{ABC}$, conciliado aos demais fatores de mercado, torna-se ferramenta importante para definição de uma estratégia de formação de preço de venda para cada ambiente, tornado o negócio sustentável.

Conclui-se que o método de custeio $\mathrm{ABC}$ mostra-se relevante para um laboratório que presta serviços de análises físico-químicas para estudos ambientais, o que apresenta gastos indiretos na razão de $86 \%$ da composição do total do custo mensurado.

Destacam-se como limitação da pesquisa a valorização dos serviços que não apresentaram demanda no período pesquisado, como: i) monitoramento de amônia; e ii) monitoramento de metais. Além disso, como o laboratório em estudo integra a estrutura de uma 
IES, alguns valores de custos alocados nesse laboratório passaram por algum critério de rateio anteriormente, utilizando métodos de custeio tradicionais, tais como, energia elétrica, telefone e valor de mão de obra de alguns funcionários. Ressalta-se, ainda, que gastos com amortização de softwares, por exemplo, não são distribuídos aos setores de laboratório, pois a IES aloca diretamente ao instituto de pesquisa, bem como depreciação e manutenção do imóvel.

Sugere-se o desenvolvimento de novas pesquisas, com a ampliação do período da coleta de dados, aplicação do método $\mathrm{ABC}$ em outras empresas do mesmo segmento, a fim de comparar os preços sugeridos nesse estudo com novos resultados e identificar possíveis fatores de mercado que possam influenciar na formação do preço de venda.

\section{Referências}

ANDRADE, M. M. Introdução à metodologia do trabalho científico. 8. ed. São Paulo: Atlas, 2007.

BORINELLI, M. L.; DOS SANTOS, M. C. P. O impacto do custeio baseado em atividades na tomada de decisão: um estudo de caso em uma Instituição Financeira. In: Anais do Congresso Brasileiro de Custos-ABC. 2014.

BRUNI, A. L.; FAMÁ, R. Gestão de custos e formação de preços: Com aplicação na calculadora HP 12C e Excel. $3^{\mathrm{a}}$ Ed. São Paulo: Atlas S.A., 2004.

CAVALCANTI, R. A. P. et al. Aplicação do método de custeamento baseado em atividade em uma clínica de radiologia odontológica: Um Estudo de Caso. In: Anais do Congresso Brasileiro de Custos-ABC. 2011.

DIEHL, A. A.; TATIM, Denise Carvalho. Pesquisa em ciências sociais aplicadas. São Paulo: Pearson Prentice Hall, 2004.

DUBOIS, A.; KULPA, L.; SOUZA, L. E. Gestão de custos e formação de preços: Conceitos, modelos e instrumentos: abordagem do capital de giro e da margem de competitividade. São Paulo: Atlas, 2006.

FERREIRA, R. J. Contabilidade de custos: teoria e questões comentadas conforme a lei das S/A e pronunciamento do CPC. Rio de Janeiro: Ferreira, 2016.

GIL, A. C. Como elaborar projetos de pesquisa. São Paulo: Atlas, 1996.

MARTINS, E. Contabilidade de custos. 9. Ed. São Paulo: Atlas, 2003.

MEGLIORINI, E. Custos: análise e gestão. São Paulo: Pearson Prentice Hall, 2007.

NAKAGAWA, M. ABC Custeio baseado em atividades. 2. ed. São Paulo: Atlas, 2001.

PRODANOV, C. C.; FREITAS, E. C.; Metodologia do trabalho científico: métodos e técnicas da pesquisa e do trabalho acadêmico. 2. ed. Novo Hamburgo, 2013.

RIBEIRO, E. N.; CAMACHO, R. R. Custeio ABC aplicado a um centro municipal de educação infantil: um estudo de caso. In: Anais do Congresso Brasileiro de Custos-ABC. 2006. 
SANTOS, J. J. Contabilidade e análise de custos: Modelo contábil, Método de depreciação, ABC - Custeio Baseado em Atividade, Análise atualizada de encargos sociais sobre salário, custos de tributos sobre compra e vendas. São Paulo: Atlas, 2011.

SANTOS, J. L. et al. Manual de contabilidade de custos. São Paulo: Atlas, 2015.

SERRATINE; K. C.; RAUPP, F. M. Proposta de implantação do custeio baseado em atividades na gestão de serviços odontológicos. Revista Catarinense da Ciência Contábil - CRCSC, Florianópolis, v.12, n.34, p.9-22, dez/mar, 2013.

TORRES, S. B.; SILVA, A. S.; MATSUMOTO, A. S. Análise para implantação do método de custeio baseado em atividade - ABC. In: Anais do Congresso Brasileiro de Custos-ABC. 2006.

YIN, R. K. Estudos de caso: planejamento e método. Porto Alegre: Bookman, 2010. 